\title{
Skin-lightening and anti-ageing effect of a food supplement containing Pinus pinaster extract
}

Yannick Piriou, Anne Sirvent, Audrey Natalizio, Frédérique Girard-ory

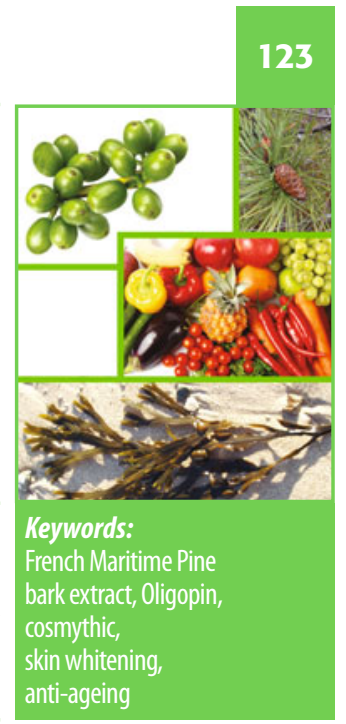

Received: 31 March 2014 / 4 July 2014

(C) The Author(s) 2014. This article is published with open access at Springerlink.com

\section{Abstract}

Oligomeric proanthocyanidins extracted from the bark of the French maritime pine Pinus pinaster (FMPBE) have been studied for a long time and demonstrated various phytopharmaceutical applications. The objectives of this study were to assess the cutaneous lightening and anti-ageing effects of FMPBE after 56 days of consumption, in healthy Asian women. The cosmetic efficacy of the product was assessed through various biometrological evaluations. This randomized, double-blind, placebocontrolled and cross-over study evidenced the safety and efficacy of the $100 \mathrm{mg}$ FMPBE supplementation from 28 days of intake: in comparison to placebo, the skin on pigmentary spots became significantly lighter and less red, skin on the face was more firm and its surface was less wrinkled. After 56 days of consumption, the skin was additionally significantly more elastic and the yellow constituent of the skin decreased on the normal skin.

Yannick Piriou $(\bowtie)$

DRT (Les Dérivés Résiniques et Terpéniques)

Purextract Route de Linxe

40560 Vielle Saint Girons, France

yannick.piriou@drt.fr

A. Sirvent, A. Natalizio, F. Girard Ory

Laboratoire Dermscan

69100 Villeurbanne - France
Taken together, these results proved that FMPBE supplementation can be considered as an innovative approach to skin whitening and anti-ageing treatments. It represents an interesting alternative to topical products and supports the skin from the inside out.

\section{Introduction}

Polyphenols are a wide range of biological molecules which play a protective role in plants. They are one of the major types of secondary metabolites and include several types of molecules, such as phenolic acids and flavonoids. Oligomeric ProanthoCyanidins (OPC) - also designated as procyanidins, leucoanthocyanidins or condensed tannins - are oligomeric flavonoids. OPCs are very common in all plants and are found in many foods (fruits, vegetables, chocolate, cider, wine and tea among others). Historically, their well known property was to complex proteins, which explains their astringent taste and their use in tanning. Further work, mainly by Masquelier in France, showed their veinotonic properties and vitamin C2 activity (allowing better intake and use of vitamin $\mathrm{C}$ by the body) $[1,2]$. Later, thanks to their strong antioxidant properties, more studies were carried out on OPCs in the nutrition and preventive medicine area [3].

OPCs extracted from the bark of the French maritime 
pine Pinus pinaster have been studied for a long time (for review, see [4, 5]). In vitro, French maritime pine bark extract (FMPBE) showed good antioxidant power, especially in the human low-density lipoprotein model [6]. A study evaluated the effect of longterm supplementation with FMPBE on rats and Syrian hamsters, especially on plasma antioxidant activity and atherosclerosis prevention [7]. FMPBE showed no toxicity and no side effects during the period of experimentation. Also, it did not have any influence on daily intake. FMPBE was absorbed and induced measurable biological activity. FMPBE improved the resistance of the heart against free radical oxidation. OPCs were more active and most probably more bioavailable than high-molecular-weight tannins and had an effective and strong protective antioxygen free radical activity. There was a significant reduction of oxidized lipids at the surface of the arteries (atherosclerotic arteries) in the group supplemented with FMPBE. FMPBE also prevents chronic UVB radiation-induced skin damage and carcinogenesis in melanin-possessing hairless mice [8].

A clinical study assessed the effect of a daily dosage of $80 \mathrm{mg}$ of FMPBE on melasma (40 mg twice a day) [9]. The results clearly showed an improvement both on skin lightening and melasma reduction after one month. In 2005, another clinical study showed good results in the evaluation of the effect of a blend of ingredients including FMPBE on skin beauty (mainly anti-wrinkle and skin elasticity) [10]. FMPBE, as shown by in vitro and in vivo testing and clinical studies, has been used in health and beauty food supplements and functional food products, providing interesting biological effects, without side effects [5]. The aim of this study was to precisely quantify the effect of FMPBE on skin beauty: whitening, anti-dark spot, anti-wrinkle and skin biomechanical properties.

\section{Materials and methods}

Experimental plan

The objectives of this study were to assess the cutaneous lightening and anti-ageing effects of a pine bark extract after 56 days of consumption in healthy women. The cosmetic efficacy of the product was assessed through various biometrological evaluations. A randomised, double-blind, placebo-controlled, cross-over design was employed for this study. It took place at Dermscan Asia (Bangkok, Thailand). The study was conducted in compliance with Good Clinical Practices.

\section{Subjects}

The minimum planned number of subjects to be analysed was 30. In order to participate in the study, women had to over 45 years old and Asian with phototype IV or V. They had to present slight wrinkles or fine lines on crow's feet and at least two pigmentation spots on face and/or hands and/or cleavage. The consumption of food supplements in the two previous months or during the study was forbidden. Subjects with an unbalanced weight or using any topical or systemic treatment likely to interfere with the assessment of the efficacy evaluation were excluded. Intensive exposure to sunlight or UV rays within the previous month or foreseen during the study were not accepted. Subject's participation in the study came into effect only after providing full informed consent. All procedures followed were in accordance with the ethical standards of the responsible committee on human experimentation (institutional and national) and with the Helsinki Declaration of 1975, as revised in 2000.

\section{Products}

Each active capsule contained (\% by weight) 50 mg of pine bark extract (17.9\%) (manufactured by DRT company, Purextract department, sold under the trade names Oligopin ${ }^{\circledR}$ and Cosmythic $\left.{ }^{\circledR}\right), 200$ $\mathrm{mg}$ maltodextrin (71.4\%) and $30 \mathrm{mg}$ magnesium stearate $(10.7 \%)$.

The active ingredient used in this study is a purified extract from FMPB ( $P$. pinaster, Aiton). It is obvious that, according to the extraction process used, one can obtain many finished products, even when using the same raw material, leading to differing 


\begin{tabular}{|lc|}
\hline Compound & Content (average, mass \%) \\
\hline Procyanidolic oligomers*, including & $65-75$ \\
Dimers & $15-20$ \\
Trimers & $15-20$ \\
Tetramers to hexamers & $30-40$ \\
\hline Catechin & $4-8$ \\
\hline Taxifoliol & $0.5-4$ \\
\hline Taxifoliol glucoside & $3-6$ \\
\hline Ferulate glucoside & $4-8$ \\
\hline Miscellaneous phenolic acids, including & $1-5$ \\
Ferulic acid & $0.1-1$ \\
Gallic acid & $0.5-3$ \\
Protocatechic acid & $0.5-3$ \\
Caffeic acid & $0.3-2$ \\
p-Coumaric acid & \\
*Procyanidolic oligomers: low-molecular-weight polymers (dimer to hexamer) of \\
catechine and epicatechine (sometimes referred to as low-molecular-weight con- \\
densed tannins).
\end{tabular}

Table 1 Chemical composition of FMPBE used in this study

chemical composition and biological activity [11]. Chemical composition of the present product has been established using different analytical techniques, including HPLC, gel permeation chromatography, mass spectrometry, NMR and chemical tests (Bate-Smith, Folin, vanillin test). According to these different methods, the composition of the product was defined (Table 1).

Each placebo capsule contained (\% by weight) 250 $\mathrm{mg}$ maltodextrin (89.3\%) and $30 \mathrm{mg}$ magnesium stearate $(10.7 \%)$. These capsules were identical in shape, smell, taste and weight to the active capsules. The dosage of $100 \mathrm{mg} /$ day of FMPBE tested in this study corresponded to less than 1/500th of the no observed adverse effect limit (NOAEL). This selected dosage (100 mg/day) was in accordance with some previous clinical studies: one clinical study demonstrated the effect of FMPBE at $80 \mathrm{mg} /$ day [12] for melasma treatment. Furumura et al. [13] used it at $100 \mathrm{mg} /$ day and Saliou et al. [14] used it at 1.10 $\mathrm{mg} / \mathrm{kg} /$ day.

\section{Trial schedule}

\section{Period 7}

Day 0: After a medical exam in order to check the inclusion and non-inclusion criteria, one zone on the back and two zones on subjects' spots (either on face, hands or cleavage) were defined. The person in charge of the medical exam and of the selection of the spots checked with the subjects that they were not some nevus or any other birthmark, to select only lentigo senile. Measurements of skin colour with a Minolta CM700d spectrophotometer were performed on the three defined areas. A skinprint was performed on one defined crow's foot in order to evaluate skin relief parameters using a Skin Image Analyser ${ }^{\circledR}\left(\right.$ SIA $\left.^{\circledR}\right)$ and QuantiRides ${ }^{\circledR}$ software. Biomechanical cutaneous properties on the defined crow's foot were measured with a Cutometer ${ }^{\circledR}$. A macrophotograph of each pigmentary spot was taken and the first treatment (active or placebo) was distributed to the subjects according to the randomisation list. Women had to take one capsule in the morning and one in the evening. A daily log was also provided in order to register any concomitant treatment or adverse events.

Day 28: the subjects came back to the laboratory with their treatment and completed a daily log. The same measurements were performed as on D0, on the same zones. The product was distributed again to the subjects as well as a new daily log.

Day 56: The subjects came back to the laboratory with their products and completed a daily log. The same measurements were performed as on D0, on the same zones.

After a wash-out period of 14 days, during which the subjects did not have to take any test products, the women came back for the second period of trial.

\section{Period 2}

Day 0: The same measurements were performed as the ones in Period 1 on D0, on the same zones (skin colour on back and pigmentary spots, biomechanical cutaneous properties and skinprint in crow's foot, macrophotographs on pigmentary spots). The second treatment (active or placebo) was distributed to the subjects according to the randomisation list. Women had to take one capsule 
in the morning and one in the evening. A daily log was also provided in order to register any concomitant treatment or adverse events.

On D28 and D56, the subjects came back to the laboratory with their treatment and completed daily log. The same measurements as on D0 in Period 1 were performed, on the same zones.

\section{Evaluation tools}

\section{CM700d spectrophotometer (Minolta)}

Skin colorimetric measurements were performed with apparatus equipped with a $3 \mathrm{~mm}$ diameter head. The device converts colours perceived by humans to a digital code composed of three parameters: $L^{*}$ (for clarity from dark to light), $a^{*}$ (for the green-to-red spectrum) and $\mathrm{b}^{*}$ (for the blue-to-yellow spectrum). $a^{*}$ and $b^{*}$ are chrominance parameters and $\mathrm{L}^{*}$ is a luminance parameter. It is therefore possible to express the differences between two cutaneous zones that appear to be the same colour in minute detail. After a calibration phase, measurements are made directly on the skin using a pulsed xenon light source and a dual beam system designed to measure the light transmitted and to correct any slight deviation. This instrument is commonly used in cosmetics and medicine to measure skin colour. The parameters $\mathrm{L}^{*}$ (luminance) and $\mathrm{b}^{*}$ (cutaneous melanin yellow colour) are studied during a lightening product study. Both parameters are exploited through the calculation of the individual typological angle, which defines the skin pigmentation degree of a subject according to the following formula: $\mathrm{ITA}^{\circ}=\left[\operatorname{Arc} \tan \left(\left(\mathrm{L}^{*}-50\right) / \mathrm{b}^{*}\right)\right] \times 180 / \pi$. The higher the ITA $^{\circ}$ is, the lighter the skin is. For pigmentary spots, the $\mathrm{a}^{*}$ parameter is also interesting to study because the "red" component is important in spots.

\section{$S I A^{\circledR}$}

Polymer silicon skinprints $\left(\right.$ Silflo $^{\circledR}$ ) were taken of the studied zones, before product use and at each time of measurement, then studied using a Skin Image Analyser $^{\circledR}\left(\mathrm{SIA}^{\circledR}\right)$. For that purpose, an oblique lighting of $35^{\circ}$ creates shadows on the replica surface.
These shadows are observed with a digital camera linked to a computer. A $1 \mathrm{~cm}^{2}$ area is studied. The digitised picture obtained is analysed in grey levels and allows assessment of different parameters of the skin surface relief. The parameters studied with the QuantiRides $^{\circledR}$ software (Monaderm, Monaco) are the total wrinkled surface (in $\mathrm{mm}^{2}$ ), and the number and the average depth (in $\mu \mathrm{m}$ ) of cutaneous microrelief furrows (depth $<55 \mu \mathrm{m}$ ), medium wrinkles (55-110 $\mu \mathrm{m}$ depth) and deep wrinkles (depth >110 $\mu \mathrm{m})$. A decrease in the microrelief furrows characterises a smoothing effect. Decreases in the medium or deep wrinkles and of the total wrinkled surface characterise an anti-wrinkle effect.

\section{SEM 575 Cutometer ${ }^{\circledR}$ (Courage \& Khazaka)}

The skin is sucked into the orifice of a probe by constant vacuum pressure (300 mbar) for $3 \mathrm{~s}$ followed by a 2 -s relaxation period. The depth to which the skin penetrates into the probe is measured by two optical prisms located at the opening of the probe's orifice. These measurements define different parameters characterising the biomechanical properties of tegument:

- Firmness (parameter R0): A decrease of this parameter means firmer skin

- Elasticity (parameters R2, R5 or R7): An increase of one of these parameters indicates more elastic skin. Parameter R6 is also representative of the state of skin elasticity: its decrease characterises more elastic skin.

- Fatigability (parameter R9): A decrease of this parameter indicates an improvement of the cutaneous state.

\section{Macrophotography}

The digital camera used was a Nikon D100 or D70. The photographs were taken in standardised, indirect light. Aperture, speed and distance of the camera were also standardised. The control of the repositioning took place directly on a data-processing screen thanks to simultaneous visualisation of the images at various times of acquisition. 


\section{Statistics}

For each studied parameter (spectrophotometer and Cutometer ${ }^{\circledR}$ measurements, wrinkle analysis) and for each product, the descriptive statistics for quantitative variables of the original data (before and after 28 days and 56 days of product use) and of the changes from baseline (Dx-D0) were computed and tabulated.

A mixed ANOVA model was fitted to the change outcome (Di-D0). The factors taken into account were product effect (as fixed with two levels), period effect (as fixed with two levels, D0 to D56 and D70 to D126), sequence effect (as fixed with two levels, product/placebo and placebo/product) and subject nested within sequence (as random effect). The LSmeans statement will be used to (i) assess the change on Di from baseline (D0) within each group of product and (ii) test whether the products differ significantly between them.

The underlying assumptions (homoscedasticity and residual normality) were checked with graphs and the Shapiro-Wilk test $(\alpha=0.01)$. In case of strong deviation, an equivalent non-parametric approach was applied. Each categorical response of the subjective questionnaire was described and tabulated by frequency and percentage $(\mathrm{N}, \%)$ at each evaluation time. No comparative analysis between products was performed. The type I error was set at $10 \%$ in a two-tailed approach and the Microsoft ${ }^{\circledR}$ Excel and $\mathrm{SAS}^{\circledR} 9.2$ software programs were used.

\section{Results}

Thirty-five women were included in the study but one subject did not come for the visit on D28 and was therefore dropped from the study. Thirty-four women, with a mean age of $60 \pm 1$ years (46-70 years), completed the study. Both treatments were very well tolerated, with no declared adverse effects after 56 days of intake.

\section{Skin colour evaluation on normal skin (back) (see Table 2)}

After 56 days of FMPBE intake, $\mathrm{L}^{*}$ and $\mathrm{ITA}^{\circ}$ parameters significantly increased $(p=0.076$ and $p=0.016$ re-

\begin{tabular}{|c|c|c|c|c|}
\hline Parameter & Change & Product & Mean $\Delta \%$ & $p$-value \\
\hline \multirow[t]{6}{*}{$\mathrm{ITA}^{\circ}$} & (D28-D0) & FMPBE & 1 & 0.058 \\
\hline & & Placebo & 1 & 0.032 \\
\hline & & FMPBE vs. placebo & & 0.838 \\
\hline & (D56-D0) & FMPBE & 7 & 0.016 \\
\hline & & Placebo & 2 & 0.203 \\
\hline & & FMPBE vs. placebo & & 0.355 \\
\hline \multirow[t]{6}{*}{$L^{*}$} & (D28-D0) & FMPBE & 0 & 0.250 \\
\hline & & Placebo & 0 & 0.129 \\
\hline & & FMPBE vs. placebo & & 0.782 \\
\hline & (D56-D0) & FMPBE & 1 & 0.076 \\
\hline & & Placebo & 0 & 0.216 \\
\hline & & FMPBE vs. placebo & & 0.653 \\
\hline \multirow[t]{6}{*}{$b^{*}$} & (D28-D0) & FMPBE & -1 & 0.113 \\
\hline & & Placebo & 0 & 0.518 \\
\hline & & FMPBE vs. placebo & & 0.493 \\
\hline & (D56-D0) & FMPBE & -1 & 0.015 \\
\hline & & Placebo & 0 & 0.928 \\
\hline & & FMPBE vs. placebo & & 0.097 \\
\hline
\end{tabular}

Table 2 Skin colour estimate changes (mean $\Delta \%$ ) on the back and comparison of products: analysis of the $\mathrm{ITA}^{\circ}$, $\mathrm{L}^{*}$ and $\mathrm{b}^{*}$ parameters

spectively), reflecting a significant lightening effect of the skin and a decrease of the cutaneous pigmentation on the back. The $b^{*}$ parameter also significantly decreased after 56 days of intake ( $p=0.0145)$, meaning that the yellow constituent of the skin was reduced. No significant modification of any of these parameters was observed after 56 days of placebo consumption.

When comparing the two treatments on D56, the FMPBE significantly decreased the yellow constituent of the skin ( $b^{*}$ parameter) compared to placebo $(p=0.097)$.

\section{Skin colour evaluation on pigmentary spots see Table 3)}

Already after 28 days of FMPBE intake, $\mathrm{L}^{*}$ and $\mathrm{ITA}^{\circ}$ parameters were significantly increased and $a^{*}$ was significantly decreased reflecting a significant lightening effect of the skin and a decrease of the red cutaneous pigmentation on spots. Regarding placebo consumption, skin appeared significantly more red on spots after 56 days of intake ( $\mathrm{a}^{*}$ parameter; $p=0.047$ ). 


\begin{tabular}{|c|c|c|c|c|}
\hline Parameter & Change & Product & Mean $\Delta \%$ & $p$-value \\
\hline \multirow[t]{6}{*}{$\mathrm{ITA}^{\circ}$} & (D28-D0) & FMPBE & 6 & 0.004 \\
\hline & & Placebo & 2 & 0.349 \\
\hline & & FMPBE vs. placebo & & 0.093 \\
\hline & (D56-D0) & FMPBE & 12 & 0.000 \\
\hline & & Placebo & 4 & 0.165 \\
\hline & & FMPBE vs. placebo & & 0.062 \\
\hline \multirow[t]{6}{*}{$L^{*}$} & (D28-D0) & FMPBE & 0 & 0.001 \\
\hline & & Placebo & 0 & 0.220 \\
\hline & & FMPBE vs. placebo & & 0.068 \\
\hline & (D56-D0) & FMPBE & 1 & 0.000 \\
\hline & & Placebo & 0 & 0.056 \\
\hline & & FMPBE vs. placebo & & 0.134 \\
\hline \multirow[t]{6}{*}{$b^{*}$} & (D28-D0) & FMPBE & 0 & 0.670 \\
\hline & & Placebo & 0 & 0.758 \\
\hline & & FMPBE vs. placebo & & 0.942 \\
\hline & (D56-D0) & FMPBE & -1 & 0.344 \\
\hline & & Placebo & 0 & 0.931 \\
\hline & & FMPBE vs. placebo & & 0.464 \\
\hline \multirow[t]{6}{*}{$a^{*}$} & (D28-D0) & FMPBE & -2 & 0.020 \\
\hline & & Placebo & 1 & 0.508 \\
\hline & & FMPBE vs. placebo & & 0.039 \\
\hline & (D56-D0) & FMPBE & -2 & 0.017 \\
\hline & & Placebo & 1 & 0.047 \\
\hline & & FMPBE vs. placebo & & 0.004 \\
\hline
\end{tabular}

Table 3 Skin color estimate changes (mean $\Delta \%$ ) on the back, on each Di from baseline (D0) and comparison of products: analysis of the parameters ITA ${ }^{\circ} \mathrm{L}^{*}, \mathrm{~b}^{*}$ and $\mathrm{a}^{*}$

After 28 days of treatment with the FMPBE and compared to the placebo, the skin was significantly lighter (ITA ${ }^{\circ}$ and $\mathrm{L}^{*}$ parameters) and less red $\left(\mathrm{a}^{*}\right)$ on pigmentary spots. It stayed significantly lighter $\left(\right.$ ITA $^{\circ}$ parameter) and less red $\left(\mathrm{a}^{*}\right)$ after 56 days of intake.

\section{Wrinkle evaluation on crow's foot skinprint (see Table 4)}

Under these study conditions, FMPBE induced the following:

- A significant decrease in the total wrinkled surface of $-15 \%$ on average ( $p=0.026$ ) after 28 days of product intake. This effect was significantly different from the placebo effect $(p=0.003)$ after 28 days and less significantly different after 56 days $(p=0.108)$.

- A significant decrease in the number of medium wrinkles of $-12 \%$ on average $(p=0.075)$ after 28 days of product intake.
- A significant decrease in the depth of deep wrinkles after 28 and 56 days of product intake of respectively $-7 \%(p=0.081)$ and $-8 \%(p=0.085)$ on average.

These results characterise an anti-wrinkle effect of FMPBE after 28 days of intake. The anti-wrinkle effect was measured in approximately $65 \%-71 \%$ of the subjects.

Fifty-six days of placebo consumption induced a significant reduction of $-2 \%$ on average in the depth of microrelief furrows ( $p=0.087$ ), meaning smoother skin, without anti-wrinkle effect. This smoothing activity was observed on $62 \%$ of the subjects.

\section{Evaluation of cutaneous biomechanical}

\section{properties on the temple (data not shown)}

After 28 days and 56 days of FMPBE intake and in comparison with the initial state:

- Skin firmness was significantly increased (mean variations of $-8 \%$ after 28 days, $p<0.001$; and $-7 \%$ after 56 days, $p<0.001)$. The improvement of skin firmness was statistically different from the placebo group ( $p=0.014$ on D28 and $p=0.014$ on D56).

- Skin elasticity was significantly improved on D56 (mean variations of $+3 \%, p=0.057$ for R2 parameter and $+4 \%, \mathrm{p}=0.066$ for $\mathrm{R} 7$ parameter).

- Skin fatigability was significantly decreased (mean variations of $-9 \%$ after 28 days, $p=0.002$ and $-9 \%$ after 56 days, $p=0.028$ ). The reduction of skin fatigability was statistically different from the placebo group on D28 ( $p=0.028)$.

No significant variation of rheological characteristics of the skin was registered with the placebo treatment.

\section{Macrophotographies}

An example of results obtained with FMPBE on pigmentary spots is presented in Fig. 1.

\section{Discussion}

Within Asia, the population 65 years and older is expected to grow dramatically over the next 50 


\begin{tabular}{|c|c|c|c|c|}
\hline Parameter & Change & Product & Mean $\Delta \%$ & $p$-value \\
\hline \multirow[t]{6}{*}{ Depth of microrelief furrows $(\mu \mathrm{m})$} & (D28-D0) & FMPBE & 0 & 0.636 \\
\hline & & Placebo & 0 & 0.782 \\
\hline & & FMPBE vs. placebo & & 0.876 \\
\hline & (D56-D0) & FMPBE & 0 & 0.71 \\
\hline & & Placebo & -2 & 0.087 \\
\hline & & FMPBE vs. placebo & & 0.173 \\
\hline \multirow[t]{6}{*}{ Number of medium wrinkles } & (D28-D0) & FMPBE & -12 & 0.075 \\
\hline & & Placebo & -8 & 0.185 \\
\hline & & FMPBE vs. placebo & & 0.741 \\
\hline & (D56-D0) & FMPBE & 0 & 0.906 \\
\hline & & Placebo & -1 & 0.904 \\
\hline & & FMPBE vs. placebo & & 0.998 \\
\hline \multirow[t]{6}{*}{ Depth of medium wrinkles $(\mu \mathrm{m})$} & (D28-D0) & FMPBE & 2 & 0.037 \\
\hline & & Placebo & 3 & 0.001 \\
\hline & & FMPBE vs. placebo & & 0.300 \\
\hline & (D56-D0) & FMPBE & 1 & 0.455 \\
\hline & & Placebo & 1 & 0.341 \\
\hline & & FMPBE vs. placebo & & 0.882 \\
\hline \multirow[t]{6}{*}{ Depth of deep wrinkles $(\mu \mathrm{m})$} & (D28-D0) & FMPBE & -7 & 0.081 \\
\hline & & Placebo & 1 & 0.880 \\
\hline & & FMPBE vs. placebo & & 0.193 \\
\hline & (D56-D0) & FMPBE & -8 & 0.085 \\
\hline & & Placebo & 0 & 0.967 \\
\hline & & FMPBE vs. placebo & & 0.292 \\
\hline \multirow[t]{6}{*}{ Total wrinkled surface $\left(\mathrm{mm}^{2}\right)$} & (D28-D0) & FMPBE & -15 & 0.026 \\
\hline & & Placebo & 13 & 0.135 \\
\hline & & FMPBE vs. placebo & & 0.003 \\
\hline & (D56-D0) & FMPBE & -9 & 0.197 \\
\hline & & Placebo & 10 & 0.275 \\
\hline & & FMPBE vs. placebo & & 0.108 \\
\hline
\end{tabular}

Table 4 Skin relief estimate changes (mean $\Delta \%$ ) on the crow's foot, on each Di from baseline (D0) and comparison of products. Parameters with no significant change are not presented here

years. As a whole, this age group is expected to grow by 314\% in Asia: going from 207 million in 2000 to 857 million by 2050 [16]. In most Asian countries, similar to the rest of the world, older women outnumber older men, particularly in the oldest age group. As people live longer, there is a growing demand for both healthcare and skincare. Many people seek cosmetic procedures to enhance or preserve their facial features in order to maintain a youthful appearance.

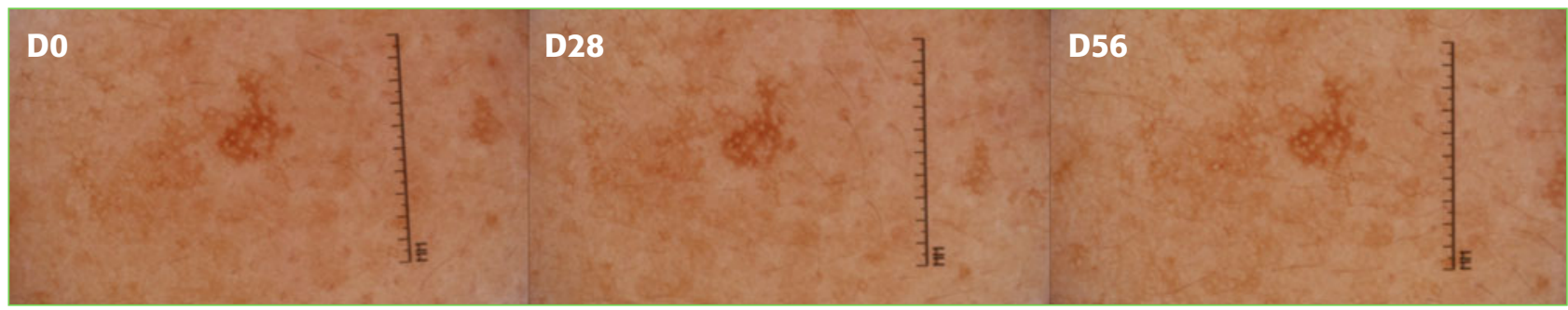

Figure 1 Example of results obtained with FMPBE. The lightening effect is visible on both the pigmentary spots and the surrounding normal skin 
The cutaneous ageing of the face is characterized, on an aesthetic level, by cumulative phenomena including cutaneous relaxation, volume reduction (notably at the cheekbones and mouth levels), the presence of wrinkles and furrows, and colour heterogeneity. These ageing manifestations are the result of the combined effects of gravity, decreased tissue elasticity, progressive bone resorption and subcutaneous fat redistribution. Pigmentary and vascular impairments are responsible for colour heterogeneity. The rate of ageing can be significantly different among different populations. Intrinsic ageing is a natural process and is genetically determined. In Asia, brown spots, clinically diagnosed as solar lentigines, are the first visible sign of ageing [17], whereas wrinkles represent the first cutaneous marks of the passing years in Europe. Cosmetic products are the most popular and most used anti-ageing treatments. They have become increasingly high-tech, acting very specifically on cellular phenomena responsible for cutaneous ageing. Topical anti-wrinkle actives have several targets: (i) at the epidermal level, they modulate keratinocyte differentiation and stimulate keratinocyte renewal; (ii) at the dermal level, they slow down dermal components' degradation and stimulate their synthesis. They also favour fibroblast renewal and water retention and maintain fundamental theme volume. Some of the well known actives used are retinol, vitamin $\mathrm{C}$, hyaluronic acid and $\alpha$-hydroxy acids. Whitening care products, leading to an overall skin lightening effect, were first aimed at Asian women. Nowadays, Western markets are also interested in these products, combating localised hyperpigmentation and dull complexion. Most whitening agents use inhibitors of tyrosinase, a key enzyme in melanogenesis, or the activity of its mediators. Topical products designed today for skin lightening mainly employ arbutin, kojic acid or azelaic acid. These ingredients are often associated with antioxidants, agents promoting desquamation and sunscreens.

Here, very strict trial conditions were set up in order to assess the skin lightening and anti-ageing effects of FMPBE after 56 days of consumption, in healthy women. This randomised, double-blind, placebo-controlled, cross-over study evidenced the efficacy of $100 \mathrm{mg}$ FMPBE supplementation from 28 days of intake: in comparison to placebo, the skin on pigmentary spots became significantly lighter and less red, skin on the face was more firm and its surface was less wrinkled. After 56 days of consumption, the skin was additionally significantly more elastic and the yellow constituent of the skin was decreased on the normal skin.

In this study, the levels of efficacy obtained thanks to oral supplementation were comparable to or better than the results that could be achieved with topical application. Furthermore, significant changes appeared already after 28 days of consumption, corresponding to rapid action. The cutaneous changes obtained in the presence of FMPBE could be the result of combined activities: it traps lipid peroxides and free radicals, and it also chelates to free iron molecules, so as to inhibit iron-induced lipid peroxidation; it non-competitively inhibits xanthine oxidase, a major generator of free radicals [3, 18]. FMPBE prevents the release of pro-inflammatory mediators regulated by oxidative stress $[14,15,19]$. As UV radiation is a major source of reactive oxygen species leading to oxidative stress, inflammation and pigmentary disorders, FMPBE is indicated to counteract UV-induced damage. The lightening effect obtained in this study on both pigmentary spots and normal skin is in accordance with previous observations on photoaged facial skin in the presence of a FMPBE supplementation [13] or UV-induced skin damage [14]. It was shown in vitro that OPCs from either FMPBE or grapeseed influence the NF-кB signalling pathway activated by UV-induced oxidative stress $[14,20]$. The firming and anti-wrinkle effects measured from 28 days of supplementation should be linked to FMPBE anti-inflammatory properties and its capacity of inhibition of enzymes degrading connective tissue structures (hyaluronidase, elastase, collagenase, gelatinase) $[21,22]$. It could be also 
linked to the enhancing action of pine OPCs on vitamin C catalyst synthesis of collagen [2].

Taken together, these results proved that FMPBE supplementation can be considered as an innovative approach to skin whitening and anti-ageing treatments. Effective skin bleaching, firming and antiwrinkle effects can be obtained already after 28 days of $100 \mathrm{mg}$ intake per day, with a high level of safety. It represents an interesting alternative to topical products and supports the skin from the inside out.

\section{Conflict of interest}

Y. Piriou is an employee of DRT, which markets the tested product. A. Natalizio, A. Sirvent and F. Girard-Ory are employees of the Dermscan group. The latter is a Contract Research Organization (CRO) that received funding from DRT in order to conduct the study.

\section{Human and Animal rights}

All procedures followed were in accordance with the ethical standards of the responsible committee on human experimentation (institutional and national) and with the Helsinki Declaration of 1975, as revised in 2000 (5).

\section{Informed consent}

Informed consent was obtained from all patients for being included in the study.

\section{References}

1. Masquelier J, Michaud J, Laparra J, Dumon MC (1979) Flavonoids and pycnogenols. Int J Vitamin Nutr Res 49:307-311

2. Michaud J, Masquelier J (1973) Quelques aspects nouveaux de la connaissance des tanins catéchiques, leurs relations avec la vitamine $\mathrm{C} 2$. Produits et problèmes pharmaceutiques 28:499-520

3. Fine AM (2000) Oligomeric proanthocyanidin complexes: history, structure and phytopharmaceutical applications. Alt Med Rev 5:144-151

4. Rohdewald P (2002) A review of the French maritime pine bark extract (Pycnogenol ${ }^{\circledR}$ ), a herbal medication with diverse clinical pharmacology. Int J Clin Pharmacol Ther 40:15-68

5. Iravani S, Zolfaghari B (2011) Pharmaceutical and nutraceutical effects of Pinus pinaster bark extract. Res Pharm Sci 6:1-11

6. Mazur A, Bayle D, Lab C et al (1999) Inhibitory effect of procyanidin-rich extracts on LDL oxidation in vitro. Atherosclerosis 145:421-422
7. Busserolles J, Gueux E, Balasinska B et al (2006) In vivo antioxidant activity of procyanidin-rich extracts from grape seed and pine (Pinus maritima) bark in rats. Int J Vitamin Nutr Res 76:22-27

8. Kimura Y, Sumiyoshi M (2010) French maritime pine bark (Pinus maritima Lam.) extract (Flavangenol ${ }^{\circledR}$ ) prevents chronic UVB radiation-induced skin damage and carcinogenesis in melanin-possessing hairless mice. Phytochem Phytobiol 86:955-963

10. Thom E (2005) A randomized, double-blind, placebo-controlled study on the clinic efficacy of oral treatment with DermaVite $^{\mathrm{TM}}$ on ageing symptoms of the skin. J Int Med Res 33:267-272

11. Assouad JL, Piriou Y (2007) Procyanidins from French Maritime Pine Bark: extraction and biological properties. NutraFoods 6:25-33

12. Shahrir M, Saadiah S, Ismail S et al. (2004) The efficacy and safety of French maritime Pine Bark extract in the form of Mscc complex actisome on melasma. Int Med J 3: 130-132

13. Furumura M, Sato N, Kusaba N, Takagaki K, Nakayama J (2012) Oral administration of French maritime pine bark extract (Flavangenol ${ }^{\circledR}$ ) improves clinical symptoms in photoaged facial skin. Clin Interv Aging 7:275-286

14. Saliou C, Rimbach G, Moini H et al (2001) Solar ultraviolet-induced erythema in human skin and nuclear factor $\kappa$ B-dependent gene expression in keratinocytes are modulated by a French maritime pine bark extract. Free Radic Biol Med 30:154-160

15. Bayeta E, Lau BHS (2001) Pycnogenol inhibits generation of inflammatory mediators in macrophages. Nutr Res 20:249-259

16. East-West Center (2002) The future of population in Asia, pp. 83-95, ISBN 0-866338-199-6

17. Hölzle E (1992) Pigmented lesions as a sign of photodamage. Br J Dermatol 127:48-50

18. Devaraj S, Vega-Lopez S, Kaul N et al (2002) Supplementation with a pine bark extract rich in polyphenols increases plasma anti-oxidant capacity and alters the plasma lipoprotein profile. Lipids 37:931-934

19. Peng Q, Wei Z, Lau BHS (2000) Pycnogenol ${ }^{\circledR}$ inhibits tumor necrosis factor $\alpha$-induced nuclear factor $\kappa \mathrm{B}$ activation and adhesion molecule expression in human vascular endothelial cells. Cell Mol Life Sci 57:834-841

20. Mantena SK, Katiyar SK (2006) Grape seed proanthocyanidins inhibit UV-radiation-induced oxidative stress and activation of MAPK and NF $\kappa B$ signaling in human epidermal keratinocytes. Free Radic Biol Med 40:1603-1614

21. Bombardelli E, Morazzoni P, Carini M et al (1997) Biological activity of procyanidins from Vitis vinifera L. Biofactors 6:429-431

22. Grimm T, Schafer A, Hogger P (2004) Antioxidant activity and inhibition of matrix metalloproteinase by metabolites of maritime pine bark extract (Pycnogenol). Free Radic Biol Med 36:811-822 